\title{
The De-beamed $\gamma$-Ray Emissions in Blazars
}

\author{
J. H. Fan $^{1,2}$ and Z. Y. Ji ${ }^{3}$ \\ ${ }^{1}$ CfA, Guangzhou University, Guangzhou 510006, China email: fjh@gzhu.edu.cn \\ ${ }^{2}$ Astron. Sci. \& Tech. Res. Lab. of Dept of Edu. of Guangdong Province, China \\ ${ }^{3}$ School of Astronomy and Space science, Nanjing University, Nanjing, China
}

\begin{abstract}
Blazars (BL Lacertae objects and flat spectrum radio quasars) are strong $\gamma$-ray emitters, the $\gamma$-ray emissions are strongly beamed. In this work, we compiled a sample of Fermi blazars with available beaming factors, $\delta_{R}$, to investigate the correlation between the $\gamma$-ray flux density, $\log f_{\gamma}$, and redshift, $\log z$ for the whole sample and the subclasses of the present sample. The analysis shows that there is no correlation between $\log f_{\gamma}$ and $\log z$ for the observed $\gamma$-ray flux density, but there are strong correlations between the de-beamed flux densities, $\log f_{\gamma}^{d b}$ and $\log z$ for the whole sample and the subclasses. Our results confirm that the $\gamma$-ray emissions are strongly beamed and imply that it is possible for one to use the radio beaming factor, $\delta_{R}$ for the beaming effect discussions in the $\gamma$-ray bands for Fermi blazars.
\end{abstract}

Keywords. galaxies:active-galaxies:BL Lacertae objects-galaxies:quasars-galaxies:jets

\section{Introduction}

Blazars are a very extreme subclass of active galactic nuclei(AGNs) showing rapid and high amplitude variability, high and variable polarization, strong and variable $\gamma$-ray emissions, and even superluminal motions etc. (Fan et al. 2013a, and reference therein). The strong $\gamma$-ray emissions in blazars suggest the existence of a relativistic beaming effect, which is discussed in the papers (see Kovalev et al. 2009; Arshakian et al. 2010; Savolainen et al. 2010; Pushkarev et al. 2010; Fan et al. 2013b,c; Giovannini, 2013; Giroletti et al. 2012; Massaro, et al. 2013a,b).

In a relativistic beaming model, the observed emission, $f^{\mathrm{ob}}$, is strongly boosted, namely, $f^{\text {ob. }}=\delta^{p} f^{\text {in }}$, here $f^{\text {in }}$ is the intrinsic emission in the source frame, $\delta$ is the Doppler factor, $p=3+\alpha$ for a moving compact source or $p=2+\alpha$ for a continuous jet (Lind \& Blandford 1985), and $\alpha$ is the spectral index $\left(f_{\nu} \propto \nu^{-\alpha_{\nu}}\right)$. The Doppler factor, $\delta$ is an important parameter, which was estimated in some papers (Ghisellini et al., 1993; Lahteenmaki \& Valtaoja, 1999; Fan et al., 2009; Hovatta et al. 2009).

In this work, we compiled a sample of 73 Fermi blazars with available Doppler factors, and investigated the correlations between the flux density and the redshift.

\section{Results and Conclusion}

For the $\gamma$-ray sources, the integral flux, $f$, in units of $\mathrm{GeV} \cdot \mathrm{cm}^{-2} \cdot \mathrm{s}^{-1}$, can be expressed in the form (Fan et al. 2013b,c)

$$
\begin{gathered}
f=N_{\left(E_{\mathrm{L}} \sim E_{\mathrm{U}}\right)}\left(\frac{E_{\mathrm{L}} E_{\mathrm{U}}}{E_{\mathrm{L}}-E_{\mathrm{U}}}\right) \ln \frac{E_{\mathrm{U}}}{E_{\mathrm{L}}}, \text { if } \alpha_{\mathrm{ph}}=2, \text { otherwise } \\
f=N_{\left(E_{\mathrm{L}} \sim E_{\mathrm{U}}\right)} \frac{1-\alpha_{\mathrm{ph}}}{2-\alpha_{\mathrm{ph}}} \frac{\left(E_{\mathrm{U}}^{2-\alpha_{\mathrm{ph}}}-E_{\mathrm{L}}^{2-\alpha_{\mathrm{ph}}}\right)}{\left(E_{\mathrm{U}}^{1-\alpha_{\mathrm{ph}}}-E_{\mathrm{L}}^{1-\alpha_{\mathrm{ph}}}\right)}
\end{gathered}
$$


here $N_{\left(E_{\mathrm{L}} \sim E_{\mathrm{U}}\right)}$ is the integral photons in the energy range of $E_{\mathrm{L}}$ and $E_{\mathrm{U}}$. In this work, $E_{\mathrm{L}}$ and $E_{\mathrm{U}}$ correspond to $1 \mathrm{GeV}$ and $100 \mathrm{GeV}$ respectively.

From the integral flux, we can get

$$
\log f=-(0.30 \pm 0.15) \log z+(0.28 \pm 0.07)
$$

with a correlation coefficient $r=-0.23$ and a chance probability of $p=5.4 \%$.

However, when we use the de-beamed integral flux densities to deal with the correlation, we have following results.

$$
\begin{gathered}
\log f^{d b^{\alpha+3}}=-(2.58 \pm 0.38) \log z-(4.3 \pm 0.17), \text { and } \\
\log f^{d b^{\alpha+2}}=-(2.08 \pm 0.30) \log z-(3.24 \pm 0.13),
\end{gathered}
$$

with correlation coefficients and the probabilities being $r=-0.63, p<10^{-4}$ for both cases.

If the Fermi blazars belong to a group, then we can expect that the flux density should follow a theoretical result as $\log f=-2.0 \log z+$ const. Our investigation for a Fermi sample with available radio Doppler factors suggests that the observed flux density does not follow that relationship, but the de-beamed ones follow that relationship with the slopes being $-2.58 \pm 0.38$ and $-2.08 \pm 0.30$ for $\alpha+3$ and $\alpha+2$ cases respectively. Our result suggests that 1) the $\gamma$-ray emissions are strongly beamed; 2) the de-beamed flux density follow the theoretical relationship for Fermi blazars; 3) the radio Doppler factors can be used to deal with the relativistic beaming effect in the $\gamma$-ray band. This is consistent with the result in our previous work, which showed that the estimated $\gamma$-ray Doppler factors are correlated with the radio Doppler factors (Fan et al. 2013d).

\section{Acknowledgements}

The work is partially supported by the National Natural Science Foundation of China (NSFC 10633010, NSFC 11173009), the Bureau of Education of Guangzhou Municipality(No.11 Sui-Jiao-Ke[2009]), Guangdong Province Universities and Colleges Pearl River Scholar Funded Scheme(GDUPS)(2009), Yangcheng Scholar Funded Scheme(10A027S), and support for Astrophysics Key Subject of Guangzhou City.

\section{References}

Arshakian, T. G., Torrealba, J., Chavushyan, V. H., et al., 2010, A\&A, 520, A62

Fan, J. H., Yang, J. H., Zhang, J. Y., et al., 2009, PASJ, 61, 639

Fan, J. H., et al. 2013a, IAUS 304, Multiwavelength AGN Surveys and Studies, this proceedings

Fan, J. H., Yang, J. H., Zhang, J. Y., et al., 2013b, PASJ, 65, 25

Fan, J. H., Yang, J. H., Liu, Y., \& Zhang, J. Y., 2013c, RAA, 13, 259

Fan, J. H., Bastieri, D., Yang, J. H., et al., 2013d, RAA, (submitted)

Ghisellini, G., et al., 1993, ApJ, 407, 65

Giovannini, G., 2013, IAUS 304, Multiwavelength AGN Surveys and Studies, this proceedings

Giroletti, M., Pavlidou, V., Reimer, A., et al. 2012, AdSpR, 49, 1320

Kovalev, Y. Y. 2009, ApJ, 707, 56

Lähteenimäki, A. \& Valtaoja, E., 1999, ApJ, 521, 493

Lind, K. R. \& Blandford, R. D., 1985, ApJ, 295, 358

Massaro, F., Giroletti, M., Paggi, A., et al. 2013a, ApJS, 207, 4

Massaro, F., D'Abrusco, R. Giroletti, M., et al. 2013b, ApJS, 208, 15

Pushkarev, A. B., Kovalev, Y. Y., \& Lister, M. L., 2010, ApJ, 722L, 7

Savolainen, T., Homan, D. C., Hovatta, T., et al., 2010, A\&A, 512A, 24 